\title{
DOCENTES UNIVERSITARIOS INCLUSIVOS: QUÉ LES CARACTERIZA Y CÓMO CONCIBEN LA DISCAPACIDAD
}

\section{Inclusive University Teachers: What Characterizes them and how do they Think about Disability}

\author{
María Nieves SÁNchez Díaz \\ Universidad de Sevilla. Departamento de Psicología Evolutiva y de la Educación \\ msdiaz@us.es \\ Beatriz Morgado Camacho \\ Universidad de Sevilla. Departamento de Psicología Evolutiva y de la Educación
}

Recepción: 12 de marzo de 2020

Aceptación definitiva: 30 de julio de 2020

Resumen: El incremento de estudiantes con discapacidad en las universidades españolas conlleva la necesidad de desarrollar prácticas docentes inclusivas que garanticen la participación y el aprendizaje de todo el alumnado. Con este trabajo se pretende: (1) conocer las concepciones que el profesorado universitario que ejerce prácticas docentes inclusivas tiene sobre la discapacidad; (2) analizar las razones que les motivan para tener en cuenta las necesidades de los estudiantes con discapacidad, y (3) describir las características que les definen. En el estudio participaron 42 docentes que fueron seleccionados como inclusivos por estudiantes con discapacidad de seis universidades públicas de diferentes comunidades autónomas de España (Andalucía, Valencia y Madrid). Estos docentes pertenecían a diferentes áreas de conocimiento y todos impartían docencia en Facultades de Ciencias de la Educación. Se siguió una metodología biográfico-narrativa, mediante el uso de entrevistas semiestructuradas. Los resultados indicaron que la mayoría del profesorado concebía la discapacidad desde el modelo social, abogando por una educación inclusiva. Entre las razones que les llevaban a responder a las necesidades de este alumnado estaban la experiencia previa con personas con discapacidad, el principio de igualdad y su responsabilidad como docente. Respecto a las características de estos docentes inclusivos, destacan la empatía, el compromiso, la flexibilidad y la vocación docente.

Palabras Clave: creencias; discapacidad; profesorado universitario; educación inclusiva; educación superior. 
АвsтRAст: The increase in the number of students with disabilities in Spanish universities implies the need to develop inclusive teaching practices that guarantee the participation and learning of all students. This work is intended: (1) to know the conceptions that the university teaching staff who carry out inclusive teaching practices have about disability; (2) to analyze the reasons that motivate them to attend to the needs of students with disabilities, and (3) to describe the characteristics that define them. The study involved 42 faculty members who were selected as inclusive by students with disabilities from six public universities in different autonomous communities of Spain (Andalusia, Valencia and Madrid). These faculty members belonged to different areas of knowledge and all of them taught in Faculties of Educational Sciences. A biographical-narrative methodology was used by using semi-structured interviews. Results indicated that the majority of the faculty conceived disability from the social, advocating for an inclusive education. Among the reasons that led them to approach to these students were previous experience with people with disabilities, the principle of equality and their responsibility as lecturers. Regarding the characteristics of these inclusive faculty members, empathy, commitment, flexibility and teaching vocation stand out.

KEYwORDs: beliefs; disability; faculty members; inclusive education; higher education.

\section{Introducción}

$\tau$ A EDUCACIÓN INCLUSIVA es un proceso que implica y compromete la transformación de las instituciones educativas para que en ellas tenga cabida todo el alumnado, sin exclusión alguna por razón de género, etnia, religión, hándicap, orientación sexual o nivel socioeconómico (Lombardi, Vukovic y Sala-Bars, 2015). Informes como la Declaración Mundial de Educación para todos (Naciones Unidas, 1990) o la Convención Internacional sobre los Derechos de las Personas con Discapacidad (Naciones Unidas, 2006) muestran avances significativos en materia de inclusión educativa. No obstante, la educación inclusiva aún sigue siendo un reto para muchas universidades. En España, la Ley Orgánica 4/2007 de universidades determina que deben garantizarse los principios de igualdad de oportunidades y no discriminación por razón de discapacidad, y que los edificios e instalaciones universitarias deben ser accesibles.

Estos cambios normativos se han ido reflejando en el modo en que se ha estudiado la discapacidad. Algunos estudios se han situado en el modelo médico de discapacidad, que atribuye las causas de esta al propio individuo (Bingham, Clarke, Michielsens y Van de Meer, 2013; Palmer y Harley, 2012). Desde este planteamiento, las personas con discapacidad solo se consideran válidas para la sociedad si se encuentran "normalizadas" (Bingham et al., 2013). Otros trabajos han partido del modelo social de discapacidad, el cual ubica las causas de la discapacidad en la sociedad. Desde este enfoque se entiende que las necesidades que presentan las personas con discapacidad provienen de la respuesta colectiva de la sociedad ante estas (Gallagher, Connor y Ferri, 2014; Oliver y Barnes, 2010). Cada vez son más frecuentes los estudios que se sitúan en el modelo ecológico, entendiendo la discapacidad como la interacción entre 
los factores internos o personales y los externos o contextuales (Céspedes, 2005; Wolfe y Riggs, 2017). Respecto a los factores contextuales, Céspedes (2005) señala que para alcanzar la inclusión de las personas con discapacidad es preciso tener en cuenta el papel que juegan los contextos directos de desarrollo (microsistemas) tales como la familia, el contexto educativo y los iguales, y la relación que se da entre los mismos (mesosistema). Igualmente, indica que ha de tenerse en cuenta la influencia que ejerce de manera más indirecta la comunidad más cercana a la persona (exosistema) y las actitudes y creencias sociales respecto a la discapacidad (macrosistema).

Desde los planteamientos de la educación inclusiva se considera la discapacidad como una riqueza y no como un problema (Sapon-Shevin, 2013) y se parte del presupuesto de que todo el alumnado, sea cual sea su condición, debe tener la oportunidad de recibir un aprendizaje de calidad y el derecho a participar en él. En este contexto cobran especial importancia los principios del Diseño Universal de Aprendizaje (DUA), que garantizan la respuesta a las necesidades de todos los estudiantes. Por tanto, diseñar las materias desde este enfoque permite promover la participación y el éxito de todos los estudiantes (Bunbury, 2018), sin la necesidad de realizar grandes ajustes para responder a necesidades concretas (Lombardi, Murray y Dallas, 2013).

El creciente número de estudiantes universitarios con discapacidad, junto con la defensa de los planteamientos de la educación inclusiva, ha dado paso a la aparición de nuevas líneas de investigación centradas en el estudio de prácticas docentes inclusivas en el ámbito de la educación superior (Hewett, Douglas, McLinden y Keil, 2018; Lipka, Forkosh y Meer, 2018; Rojas, Haya y Susinos, 2016; Yssel, Pak y Beilke, 2016). Estos estudios se han centrado en el análisis de las creencias, conocimientos, diseños y acciones orientadas a ofrecer una educación de calidad para todo el alumnado sin excepción (Florian, 2014; Gale y Mills, 2013). Los planteamientos de la educación inclusiva se sustentan en los principios de la pedagogía inclusiva, que ha sido identificada como una aproximación pedagógica que favorece el camino hacia una universidad accesible y pensada para todos y todas. Los trabajos que analizan la pedagogía inclusiva en la enseñanza universitaria son escasos, entre los que se pueden citar los estudios de Riddell, Edward, Weedon y Ahlgren (2010) o Riddell y Weedon (2009).

\section{La situación del alumnado con discapacidad en la universidad}

Como se ha señalado en líneas anteriores, en los últimos años ha aumentado progresivamente el número de estudiantes universitarios con discapacidad. En concreto, los datos de la Fundación Universia (2018) indican que en el curso 2017/2018 había un total de 21.435 estudiantes con discapacidad en las universidades españolas, lo que suponía un incremento del 3,1 \% con respecto al curso anterior. Esta realidad ha llevado en los últimos años a las universidades a realizar grandes esfuerzos en pro de la inclusión social y académica del alumnado con discapacidad. No obstante, aún siguen estando presentes barreras muy diversas que dificultan la vida académica de estos estudiantes (Garabal-Barbeira, Pousada, Espinosa y Saleta, 2018; Mutanga, 2018), resultando, a veces, la universidad un contexto de segregación y exclusión para 
ellos (Langørgen y Magnus, 2018). Entre las barreras que dificultan la inclusión educativa de los estudiantes con discapacidad en la universidad, se encuentran barreras físicas, como la propia inaccesibilidad de los espacios (Moriña y Morgado, 2018); de acceso al currículum, como la falta de formación e información del profesorado en materia de discapacidad y su desconocimiento sobre los posibles ajustes razonables que permiten responder a las necesidades de este alumnado (Paz, 2018); actitudinales, como la presencia de actitudes negativas de la comunidad universitaria hacia la discapacidad (Black, Weinberg y Brodwin, 2014;); y políticas, como la ausencia de políticas inclusivas reales que se materialicen en prácticas inclusivas, y garanticen la inclusión educativa y social del alumnado con discapacidad (López-Melero, 2011).

Con respecto a las barreras actitudinales, la actitud de los docentes juega un importante papel en la inclusión del alumnado con discapacidad en la universidad. En general, el profesorado muestra una buena actitud hacia estos estudiantes (Martins, Borges y Gonçalves, 2018). No obstante, en ocasiones hay docentes que no se muestran sensibles a sus necesidades y no siempre ofrecen respuestas ajustadas a las mismas (Becker y Palladino, 2016). En este sentido, Hamour (2013) afirma que el éxito de este alumnado se ve afectado significativamente por la actitud del profesorado, así como por su disposición a realizar los ajustes necesarios para cubrir sus necesidades. Estudios recientes resaltan la actitud del profesorado como un elemento clave para poder conseguir que el proceso de enseñanza-aprendizaje sea realmente inclusivo (Fullarton y Duquette, 2016).

En los últimos años está creciendo el interés por conocer cómo es la participación y qué caracteriza las experiencias del alumnado con discapacidad en la universidad (Fuller, Riddell y Weedon, 2009; Riddell, Tinklin y Wilson, 2005). No obstante, son muchos los interrogantes que quedan por responder. Autores como Gibson (2015) o Weedon y Riddell (2016) afirman que la universidad aún no ha adoptado las estrategias suficientes para incluir al alumnado con discapacidad y aún existen dificultades para el acceso y la permanencia en la universidad de este alumnado (SanahujaGavaldà, Muñoz-Moreno y Gairín-Sallán, 2020). En este sentido, se ha encontrado que los docentes que abogan por una educación inclusiva ofrecen oportunidades de aprendizaje a todo el alumnado, facilitando, por tanto, la inclusión del alumnado con discapacidad (De los Santos y Maxwell, 2018). No obstante, en muchas ocasiones las respuestas que se ofrecen para cubrir las necesidades de este alumnado suelen ser de carácter más reactivo que proactivo (Moriña, López-Gavira y Molina, 2014), lo que a veces implica que la atención no sea lo suficientemente eficaz ni inmediata.

Con respecto a las características personales y docentes del profesorado inclusivo, son escasos los trabajos que se han centrado en describir las características de los docentes inclusivos en el contexto universitario. No obstante, existen algunos datos que permiten conocer ciertas características personales y profesionales del profesorado que facilitan, la inclusión educativa y social de los estudiantes con discapacidad. Entre las características personales, el propio alumnado con discapacidad destaca la actitud comprensiva por parte del profesorado, la flexibilidad ante sus necesidades, la confianza mutua y la cercanía (Dalmau-Montalà, Sala-Bars y Llinares-Fité, 2015; Fuller, Healey, Bradley y Hall, 2004; Kioko y Makoelle, 2014). Por su parte, el profesorado 
resalta la flexibilidad y la actitud comprensiva (Kioko y Makoelle, 2014; Reupert, Hemmings y Connor, 2010).

En cuanto a las características profesionales, el alumnado con discapacidad señala que son docentes que se caracterizan por usar diferentes medios, formas y canales para transmitir la información y los contenidos de aprendizaje, por adaptar estos contenidos a las necesidades del alumnado y por estar informados y formados en materia de discapacidad (Dalmau-Montalà et al., 2015; Fuller et al., 2004; Kioko y Makoelle, 2014; Molina, Perera, Melero, Cotán y Moriña, 2016). El profesorado, al igual que los estudiantes con discapacidad, destaca, entre las características profesionales de un docente inclusivo, la buena disposición para proveer las herramientas necesarias para facilitar el aprendizaje a su alumnado, el uso de metodologías diversas y de variedad de canales para transmitir los contenidos de aprendizaje, y haber recibido información y formación sobre las diferentes discapacidades y sobre cómo ayudar a este alumnado (Kioko y Makoelle, 2014; Reupert et al., 2010).

Como se puede apreciar, la mayor parte de las características, que tanto el alumnado como el profesorado universitario identifican en el docente inclusivo, no están exclusivamente vinculadas al hecho de tener o no una discapacidad. Por tanto, son características que benefician a todo el alumnado y no solo a quien tiene una discapacidad (Moriña y Carballo, 2018).

El propósito de este estudio es analizar las creencias y los conocimientos del profesorado sobre discapacidad. Concretamente, se persigue conseguir los siguientes objetivos:

1. Conocer las concepciones que el profesorado universitario tiene sobre la discapacidad.

2. Explorar las razones que llevan al profesorado a abordar las necesidades que presenta el alumnado con discapacidad.

3. Describir las características que definen a los docentes como inclusivos.

\section{Método}

Este trabajo forma parte de un proyecto más amplio financiado por el Ministerio de Economía y Competitividad de España, titulado Pedagogía Inclusiva en la Universidad: Narrativas del Profesorado (MINECO, ref. EDU2016-765887-R), en el que participaron 119 docentes de todas las áreas de conocimiento de diez universidades públicas españolas. El objetivo de este estudio era conocer las creencias y los conocimientos que el profesorado universitario que desarrolla prácticas educativas inclusivas tiene sobre discapacidad, cómo contemplan esa realidad en sus proyectos docentes y qué acciones desarrollan en el aula para facilitar la inclusión.

Para este trabajo se ha seleccionado exclusivamente al profesorado que impartía docencia en las distintas facultades de Ciencias de la Educación. En particular, se persiguió analizar las creencias y los conocimientos que tenían estos docentes sobre la discapacidad. Se ha seguido una metodología cualitativa. Concretamente, se usó el método biográfico-narrativo, con el que se pretende dar voz al profesorado inclusivo 
que ha sido seleccionado por su propio alumnado con discapacidad. Así, las preguntas de investigación que guiaron este análisis fueron las siguientes: ¿Qué entiende el profesorado por discapacidad? ¿Cuáles son las razones por las que el profesorado universitario atiende a su alumnado con discapacidad? ¿Cómo se define el profesorado inclusivo?

\subsection{Participantes}

En este estudio participaron un total de 42 docentes de las facultades de Ciencias de la Educación, pertenecientes a seis universidades públicas españolas de tres comunidades autónomas (tres de Andalucía, dos de la Comunidad Valenciana y una de la Comunidad de Madrid). Para acceder a los participantes, por un lado, se contactó con los servicios de atención a estudiantes con discapacidad de las seis universidades, los cuales informaron a los estudiantes sobre el proyecto de investigación y solicitaron su colaboración. Y, por otro, se utilizó la técnica bola de nieve (Dusek, Yurova y Ruppel, 2015). En concreto, se pidió a distintos miembros de la comunidad universitaria (docentes, alumnado y otros agentes) la difusión del proyecto entre el alumnado con discapacidad.

A los estudiantes, se les pidió que seleccionaran al profesorado que había facilitado su inclusión educativa en la universidad. Partiendo de las propuestas de Gale y Mills (2013) y Florian (2014), se articuló un marco de análisis basado en una propuesta pedagógica que abarca cuatro dimensiones: creencias, conocimientos, diseños y acciones. Para este fin, se proporcionó a los estudiantes un conjunto de criterios que debía cumplir este profesorado inclusivo: cree en las posibilidades de todos los estudiantes; facilita los procesos de aprendizaje; su enseñanza es activa, utilizando diferentes estrategias metodológicas de enseñanza; muestra preocupación por el aprendizaje de sus estudiantes; muestra flexibilidad, con voluntad de ayudar; motiva a los estudiantes; mantiene relaciones cercanas y favorece las interacciones entre estudiantes; te hace sentir que eres importante, que eres uno más en el aula; permite que los estudiantes participen en la clase y construir el conocimiento juntos; la comunicación que mantiene contigo y con tus compañeros es horizontal.

Finalmente, estos estudiantes ofrecieron datos (nombre y filiación) del profesorado que había facilitado su inclusión educativa y que desarrollaba prácticas educativas inclusivas en sus aulas. Se contactó con estos docentes, que enseñaban en las facultades de Ciencias de la Educación, a través del correo electrónico, donde se les presentaba el estudio y se solicitaba su participación. Se contactó con un total de 65 docentes, de los cuales, finalmente, participaron 42. Tanto al alumnado como al profesorado participante en el estudio se les garantizó el anonimato en todo el proceso de investigación, atendiendo a las directrices planteadas en la Ley Orgánica 3/2018, de Protección de Datos Personales y garantía de los derechos digitales.

Con respecto al perfil de los participantes, su edad en el momento del estudio oscilaba entre 33 y 59 años, con un promedio de 41,2 años (DT $=7,18)$. Diecisiete eran hombres $(40,5 \%)$ y 25 mujeres $(59,5 \%)$. La experiencia docente de los participantes 
osciló entre los 7 y los 32 años, con un promedio de 15,8 años (DT = 3,54). Los participantes pertenecían a diferentes áreas del conocimiento: ciencias sociales (40\%), arte y humanidades $(31 \%)$, ciencias de la salud (17\%) y ciencias (12\%).

\subsection{Instrumentos}

Se diseñaron dos entrevistas semiestructuradas ad-hoc para el estudio. La primera se centró en el análisis de las creencias y conocimientos sobre discapacidad y la segun$\mathrm{da}$, en el diseño de los proyectos docentes y en las acciones que desarrollaban en el aula para facilitar la inclusión. Previamente a su aplicación, dichos instrumentos fueron pilotados con 17 docentes universitarios no participantes en el estudio. Todas sus consideraciones o sugerencias se analizaron y se discutieron durante tres encuentros que mantuvo el equipo investigador.

En este trabajo, concretamente, se analizaron los resultados obtenidos con la primera entrevista, relativos a las creencias y conocimientos sobre discapacidad del profesorado que ejerce prácticas inclusivas. Entre las preguntas que se realizaron estaban: ¿Qué es para ti la discapacidad? ¿Cómo te definirías como docente? ¿Qué crees que es lo que te ha llevado a interesarte y a trabajar por la inclusión de los estudiantes con discapacidad? ¿Cuáles crees que son las características, personales y profesionales, fundamentales que debe tener el profesorado que desarrolla educación inclusiva y da una respuesta educativa de calidad a todo el alumnado?

\subsection{Procedimiento}

Las entrevistas fueron realizadas por los miembros del equipo de investigación formados previamente para ello. La mayoría de las entrevistas se hicieron de manera presencial $(\mathrm{n}=34)$, seis se realizaron a través de la plataforma Skype y dos por vía telefónica. Todas ellas fueron grabadas en audio y tuvieron una duración de entre 1 hora y 1 hora y 30 minutos. Finalmente, todas las entrevistas fueron transcritas de manera literal.

La recogida de datos se llevó a cabo a lo largo de siete meses. El análisis de los datos se elaboró a partir de un sistema de categorías y códigos, siguiendo las propuestas de Creswell (2009) y Gale, Heath, Cameron, Rashid y Redwood (2013). En concreto, se desarrolló una codificación progresiva (Huber, 2004). A partir del guion de entrevista, se creó un sistema de categorías muy amplio, que fue completándose posteriormente en un segundo momento de codificación de las entrevistas, mediante el cual fueron apareciendo nuevos códigos (para más información véase el trabajo de Moriña, Sandoval y Carnerero, 2020). Concretamente, en este trabajo se presentan los resultados correspondientes a la dimensión de Creencias y a las categorías Concepciones sobre discapacidad (Códigos 1.1. Discapacidad-Ideas que el profesorado tiene sobre discapacidad, impresiones evocadas en el profesorado ante la palabra discapacidady 1.2. Motivaciones -Creencias sobre lo que mueve al profesorado a interesarse por 
saber las necesidades y dar respuesta a los estudiantes con discapacidad-) y Rol docente y actitudes (Código 1.3. Características docentes -Características que definen a los participantes como docentes-). Todas las entrevistas fueron analizadas y codificadas por más de un miembro del equipo investigador, para garantizar la coherencia y el consenso en el posterior proceso de análisis. Se extrajeron los resultados utilizando el software de IBM MaxQDA, en su versión 12.

\subsection{Cuestiones éticas}

Para garantizar los derechos de los participantes en esta investigación, se les proporcionó, previamente a la realización de las entrevistas, un documento de Consentimiento Informado, elaborado según el capítulo II del Real Decreto 1720/2007, de 21 de diciembre, donde se les aseguraba que los datos ofrecidos serían tratados de manera confidencial y anónima. En dicho documento se les informó que, si en algún momento de la investigación decidían abandonar el proceso de participación, sus datos serían excluidos de esta. Asimismo, los nombres de los participantes fueron sustituidos por números.

\section{Resultados}

El primer objetivo de esta investigación era conocer qué entendían los participantes por discapacidad. Los resultados encontrados indican que los docentes tenían una concepción homogénea sobre dicho concepto. Solo dos (4,8 \%) participantes del área de Ciencias (Química Orgánica), se situaban en un modelo médico de la discapacidad. El resto, bien se situaban en el modelo social de discapacidad ( $\mathrm{n}=26 ; 61,9 \%$ ), bien defendían explícitamente la necesidad de que todo el alumnado, sin excepción, se beneficie de una enseñanza de calidad y una participación plena ( $n=14 ; 33,3$ \% ).

Quienes partían del modelo de déficit o médico para definir el término de discapacidad situaban las causas de la misma en el propio individuo y la concebían en términos de déficit, dificultades y limitaciones.

La idea que se me viene es alguien con una limitación y con unas necesidades específicas para poder hacer la vida normal. Una discapacidad visual, auditiva... siempre se me viene alguien que necesita un extra, que con lo cotidiano no es capaz de llevar la vida que nosotros llevamos, ¿no? (Docente 30)

Otros docentes, sin embargo, entendían la discapacidad desde un modelo social, situando las causas de la misma en aspectos externos al individuo, tales como los prejuicios sociales y las barreras y déficits que presentaban los entornos sociales.

A mí la discapacidad me produce mucha reflexión porque me parece que es un tema que se ha utilizado para dejar fuera de la sociedad a mucha gente por sus características 
físicas o psicológicas. A mí lo que me viene es preocupación... porque me parece que la sociedad que estamos construyendo no está preparada para aceptar la diversidad... Entonces, me preocupa, pero no me preocupa por la persona, me preocupa por las condiciones sociales, porque creo que aún no estamos preparados para eso. (Docente 24)

Finalmente, algunos participantes entendían el concepto de discapacidad como un concepto que engloba a personas con diferentes capacidades y lo relacionaban con conceptos tales como igualdad, equidad y retos.

Pues diversidad, inclusión, democracia, equidad... (Docente 10)

A mí me viene a la cabeza reto. No sé si será porque yo también tengo una discapacidad, pero... en el sentido de que es un reto para el estudiante, que es diferente, es un reto para ti, que tienes que empezar a saber gestionar esas necesidades diversas, y que, en un primer momento, lo que te puede generar y que he visto que a veces genera es miedo. Miedo a tu propia incompetencia, miedo al no estar a la altura a lo mejor de lo que se espera de ti. (Docente 39)

Con el segundo objetivo de este trabajo se perseguía conocer las razones que llevaban al profesorado a abordar las necesidades de los estudiantes con discapacidad. Los resultados encontrados muestran que los participantes ofrecían argumentos y razones muy diversas para justificar su respuesta a las necesidades de estos estudiantes. Algunos $(\mathrm{n}=10 ; 23,8 \%)$ coincidían en destacar que lo que les llevaban a preocuparse por este alumnado era el hecho de haber tenido una experiencia previa con estudiantes o con amigos o familiares con discapacidad.

Hasta que tú no te topas con un alumno que es tu alumno, es tu asignatura, no eres consciente, de verdad, los puedes ver por el campus, en el centro, pero no te imaginas lo dura que puede ser la vida. (Docente 42)

Otros docentes $(\mathrm{n}=8 ; 19 \%)$ indicaban que sus intereses estaban íntimamente ligados a su formación, su perfil docente o investigador.

Bueno pues, en mi propia profesión, yo soy psicóloga de formación y bueno pues, mi propia disciplina y trabajo me hace ser sensible a toda la diversidad y a todos los colectivos minoritarios, incluido el alumnado con discapacidad. (Docente 29)

Otra razón que les hacía preocuparse y responder a las necesidades de este alumnado era el principio de igualdad $(n=25 ; 59,5 \%)$. Principio que les llevaba, de una parte, a esforzarse para ofrecer una enseñanza ajustada a las necesidades de todo su alumnado, con independencia de su origen, sus características o si presentaba o no una discapacidad. Y, por otra, a hacer referencia en sus argumentos a la atención a la diversidad, integración e inclusión de todo el alumnado.

Se me viene a la cabeza la idea de diversidad, de diferencia. Igual alguna necesidad especial, pero ya te digo, que todos en un momento determinado necesitamos una ayuda especial y no nos calificarían de discapacitados. Esa es la idea, de diferencia. (Docente 33) 
En este mismo sentido, muchos docentes indicaron que ellos no tenían un interés especial o concreto hacia el alumnado con discapacidad, sino que se preocupaban por tener en cuenta los derechos e intereses de todos sus estudiantes.

Porque creo que todos tienen el mismo derecho, incluso personas que no ven, en una asignatura que se basa en la visión, como es la mía, me preocupé en organizarle unas prácticas inclusivas, y decirle, "bueno, pues tú vas a tocar esto", por lo menos tocas, aunque no... (Docente 1 )

Además, señalaban que el hecho de considerar los intereses de los estudiantes era una obligación y una responsabilidad que tenían como docentes.

Pienso que todos los alumnos tienen derecho a su educación, a llevar sus proyectos a cabo, y como profesores creo que tenemos obligación y ética de acompañar a esos alumnos a su consecución, por lo tanto, no es un interés especial por la discapacidad, es un interés por el alumno. (Docente 35)

Con el tercer y último objetivo de este estudio, se perseguía conocer cómo se describían los participantes a sí mismos como docentes. Los resultados de este estudio indicaron que para definirse a sí mismos como docentes se basaban en características tales como el compromiso, la cercanía, gran implicación, flexibilidad y exigencia. Además, consideraban que eran docentes que se esforzaban por despertar en su alumnado una motivación intrínseca o el interés por aprender, su implicación en el proceso de aprendizaje y el valor por el respeto $(\mathrm{n}=33 ; 78,6 \%)$.

Yo... me implico mucho en todo lo que hago, totalmente, entonces, si tengo que hacerlo, lo hago, me da igual las dificultades que tenga, me busco soluciones para todo. Entonces... creo que soy una profesora comprometida, coherente, no sé, cercana, también soy muy cercana con los alumnos. (Docente 1)

Involucrarlos, yo soy mucho de involucrarlo... yo suelo invitar a la reflexión y que lo cuestionen todo. Empatía, eso, interés y un poco la flexibilidad. (Docente 35)

La mitad de los docentes $(\mathrm{n}=21 ; 50 \%)$, además, destacaba entre las características que les definían la empatía, la comunicación, el compromiso con el alumnado y la docencia, y su preocupación por innovar, por ofrecer contenidos aplicados, que la asignatura fuera accesible y que el alumnado se sintiera respetado.

Yo creo que soy cercana, intento ser lo más clara posible, lo más práctica y, sobre todo, que intento trasmitir entusiasmo por la profesión y sensibilidad. Y, además, empática. (Docente 25)

Intento, todos los años innovar, aportar algo nuevo, ofrecerles la versión aplicada... ¿sabes? (Docente 30)

Asimismo, algunos ( $\mathrm{n}=11 ; 26,2 \%$ ) se definían como personas con gran vocación por la docencia y muy motivadas para buscar respuestas a las demandas que surgen en el aula. 
Lo primero que hago es que me tomo mi trabajo como una cosa apasionante, a mí no me parece que esto sea un trabajo, sino mi hobby, o uno de mis hobbies. Y yo creo que eso se lo transmito a los alumnos. (Docente 39)

Otros ( $\mathrm{n}=7 ; 16,66 \%)$, se consideraban personas muy críticas con su propia práctica docente, lo que les llevaba a estar reflexionando constantemente sobre su actuación docente.

Siempre entiendo que hay formas de mejorar mi práctica docente... Eso sí que creo que es una actitud del trabajo en general y del trabajo en educación inclusiva o con atención a la discapacidad fundamental; el cuestionamiento constante de la propia práctica para la mejora. (Docente 12)

Y una minoría ( $\mathrm{n}=3 ; 7,1 \%$ ) indicó que incluso se preocupaba por la felicidad de sus estudiantes y por despertar en estos ese sentimiento.

Leí mucho sobre la felicidad de los alumnos y esto está relacionado con la progresión, y vi que además de la progresión, para nosotros, es muy importante crear cosas que no existen... yo creaba tareas para las características de mis alumnos o de mi materia. (Docente 5)

\section{Discusión y conclusiones}

Los resultados obtenidos con este trabajo permiten extraer conclusiones muy interesantes con respecto a los objetivos planteados. Permiten conocer cómo el profesorado universitario concibe la discapacidad, qué les lleva a responder a las necesidades del alumnado con discapacidad y cómo se describen a sí mismos como docentes inclusivos.

En cuanto a las concepciones que tiene el profesorado sobre la discapacidad, se ha podido comprobar que existe heterogeneidad entre los participantes. A diferencia de Martins et al. (2018), que encontraron que la mayoría del profesorado concibe la discapacidad desde el modelo médico, los participantes de este estudio presentan una visión heterogénea. En concreto, una minoría se sitúa en el modelo médico y el resto parte del modelo social de discapacidad o aboga por la educación inclusiva. Estos datos permiten afirmar que se están experimentando cambios en el modo en que se acoge la discapacidad en el ámbito de la educación superior. En este sentido, se ha encontrado que la formación en materia de discapacidad promueve una concepción de esta basada en el modelo social de discapacidad y en la defensa de que todo el alumnado, sin excluir a nadie, se beneficie de una enseñanza de calidad (Carballo, Morgado y Cortés-Vega, 2019).

Se ha encontrado que el modelo médico está más presente en disciplinas del área de ciencias de la salud (Rosa, Bogart, Bonnett, Estill y Colton, 2015) y que el modelo social de discapacidad y la defensa de la educación inclusiva es más visible en áreas de ciencias sociales (Zhang, Rosen, Cheng y Li, 2018). Sin embargo, los resultados de 
este trabajo indican que el modelo social y los planteamientos de educación inclusiva están cada vez más presentes en el profesorado universitario, con independencia del área de conocimiento a la que pertenecen. Encontrándose solo dos docentes del área de ciencias con concepciones vinculadas al modelo médico.

Otro aspecto de interés es la motivación del profesorado para responder a las necesidades del alumnado con discapacidad. Los datos de este estudio indican que las razones que mueven al profesorado a desarrollar prácticas inclusivas en sus aulas son muy diversas. Entre ellas, los docentes hacen referencia a cuestiones tales como la experiencia previa con otras personas con discapacidad (alumnado, amistades, familiares), la afinidad con su perfil profesional, el principio de igualdad y al cumplimiento con las tareas docentes y la normativa universitaria relativa a la atención del alumnado. Estas razones les llevan a desarrollar acciones que favorecen una práctica docente inclusiva, es decir, basadas en los pilares de la educación inclusiva, que considera la diversidad como una oportunidad y defiende el derecho de todo estudiante a recibir una educación de calidad, tal como sostienen autores como Durán y Giné (2011) o Sapon-Shevin (2013).

Con respecto a las características que definen a los participantes como docentes, el profesorado entrevistado destaca, entre sus características personales, la empatía, la habilidad comunicativa y la vocación docente. Características que a su vez consideran que debe tener el profesor inclusivo ideal. Además, se describen a sí mismos como personas cercanas, comprometidas con su trabajo y flexibles, cualidades similares a las que encontraron en sus trabajos Fuller et al. (2004), Kioko y Makoelle (2014) y Reupert et al. (2010).

Los participantes, además, hacen hincapié en otras características que les definen como profesionales. Entre ellas, destacan la formación docente como un aspecto clave para poder desarrollar prácticas inclusivas. Igualmente, indican que es importante estar informados acerca de las características de su alumnado (tipo de discapacidad, necesidades y ajustes necesarios). Estos resultados van en la misma línea de lo que plantean Dalmau-Montalà et al. (2015), Kioko y Makoelle (2014), Molina et al. (2016) y Reupert et al. (2010), quienes afirman que tanto la formación docente como la información sobre las distintas discapacidades y necesidades del alumnado son elementos que deben estar presentes en el profesorado inclusivo ideal. Todo ello permite resaltar la necesidad de que las universidades ofrezcan programas de formación específicos para su profesorado, tal y como plantean Hopkins, Round y Barley (2018).

A modo de conclusión, e imaginando el perfil que caracteriza a un docente inclusivo, se subraya la presencia de características personales tales como la empatía, la comunicación (incluyendo un trato cercano y un proceso de escucha hacia el alumnado), la flexibilidad y el compromiso con el alumnado y con la docencia; y de características profesionales como la vocación docente, formación, utilización de ejemplos aplicados y adaptados a la realidad del alumnado, reflexión constante sobre su práctica docente y preocupación por el bienestar y felicidad de su alumnado. Se trata, por tanto, de un abanico de características que, sumadas a la concepción de la discapacidad basada en el modelo social y en la educación inclusiva, servirán para que tanto el alumnado con discapacidad como el resto de estudiantes se encuentren en un ambiente de aprendizaje 
positivo, cómodo y agradable, que, en definitiva, favorezca la educación inclusiva en el ámbito de la educación superior.

Con respecto a las limitaciones de este estudio, una de ellas tiene que ver con la muestra, ya que los resultados se centran en conocer las concepciones y las características del profesorado universitario que imparte docencia en Ciencias de la Educación y que pertenecen a las áreas de Ciencias, Ciencias Sociales, Ciencias de la Salud y Arte y Humanidades, sin estar representadas otras áreas de conocimiento. El proceso de recogida de datos ha sido un proceso lento y costoso, dado que hubo que contactar primero con el alumnado con discapacidad para solicitarles los datos del profesorado que les había facilitado su inclusión educativa. A pesar de ello, el proceso ha garantizado acceder a un profesorado que verdaderamente ejerce prácticas educativas inclusivas y, por tanto, permite conocer ejemplos de buenas prácticas para favorecer la educación inclusiva en el ámbito de la educación superior. Por esto, sería recomendable que en futuros estudios se contemplen las voces del profesorado de las diferentes áreas de conocimiento, así como que se incluya a otros informantes, tales como el propio alumnado con discapacidad e incluso a estudiantes de otros colectivos minoritarios. Igualmente, sería enriquecedor conocer la posible relación entre las prácticas inclusivas y otras variables como la edad, el género, la experiencia docente, los recursos existentes en las universidades o la formación en atención a la diversidad. Todo ello, permitiría obtener una visión más amplia y detallada sobre las prácticas docentes inclusivas en el ámbito de educación superior.

\section{Referencias bibliográficas}

Becker, S. y Palladino, J. (2016). Assessing faculty perspectives about teaching and working with students with disabilities. Journal of Postsecondary Education and Disability, 29(1), 65-82. Recuperado de https://files.eric.ed.gov/fulltext/EJ1107476.pdf

Bingham, C., Clarke, L., Michielsens, E. y Van de Meer, M. (2013). Towards a social model approach? British and Dutch disability policies in the health sector compared. Personnel Review, 42(5), 613-637. https://doi.org/10.1108/PR-08-2011-0120

Black, R., Weinberg, L. y Brodwin, M. (2014). Universal Design for instruction and learning: a pilot study of faculty instructional methods and attitudes related to students with disabilities in Higher Education. Exceptionality Education International, 24(1), 48-64. Recuperado de https://ir.lib.uwo.ca/cgi/viewcontent.cgi?article=1030\&context=eei

Bunbury, S. (2018). Disability in higher education-do reasonable adjustments contribute to an inclusive curriculum? International Journal of Inclusive Education, 1-16. https://doi.org/1 0.1080/13603116.2018.1503347

Carballo, R., Morgado, B. y Cortés-Vega, M. D. (2019). Transforming faculty conceptions of disability and inclusive education through a training program. International Journal of Inclusive Education. https://doi.org/10.1080/13603116.2019.1579874

Céspedes, G. M. (2005). La nueva cultura de la discapacidad y los modelos de rehabilitación. Aquichan, 5(1), 108-113. Recuperado de https://aquichan.unisabana.edu.co/index.php/ aquichan/article/viewArticle/64/133\&

Creswell, J. (2009). Research design. Qualitative, quantitative and mixed methods approaches. California: Sage Publications. 
Dalmau-Montalà, M., Sala-Bars, I. y Llinares-Fité, M. (2015). Análisis de la formación universitaria recibida por los titulados universitarios españoles con discapacidad auditiva en la adquisición de las competencias profesionales. [Analysis of undergraduate training given to students with hearing impairment in their acquisition of professional competences]. Siglo Cero, 46(3), 255, 25-46. http://dx.doi.org/10.14201/scero20154632746

De los Santos, S. y Maxwell, G. (2018). Meeting the needs of students with disabilities at Southern Texas University from a faculty perspective. Research in Higher Education Journal, 34, 1-11. Recuperado de https://files.eric.ed.gov/fulltext/EJ1178440.pdf

Durán, D. y Giné, C. (2011). La formación del profesorado para la educación inclusiva: un proceso de desarrollo profesional y de mejora de los centros para atender a la diversidad. Revista Latinoamericana de Educación Inclusiva, 5(2), 153-170. Recuperado de https:// educrea.cl/wp-content/uploads/2017/07/DOC2-formacion-profesorado-educacion-inclusiva-1.pdf

Dusek, G. A., Yurova, V. y Ruppel, C. P. (2015). Using social media and targeted snowball sampling to survey a hard-to-reach population: a case study. International Journal of Doctoral Studies, 10, 279-299. doi:10.28945/2296.

FLORIAN, L. (2014). What counts as evidence of inclusive education? European Journal of Special Needs Education, 29(3), 286-294. https://doi.org/10.1080/08856257.2014.933551

Fullarton, S. y Duquette, C. (2016). Experiences of students with learning disabilities in Ontario universities: a case study. International Journal of Special Education, 31(1), 55-66.

Fuller, M., Healey, M., Bradley, A. y Hall, T. (2004). Barriers to learning: a systematic study of the experience of disabled students in one university. Studies in Higher Education, 29(3), 303-318. https://doi.org/10.1080/03075070410001682592

Fuller, M., Riddell, S. y WeEdon, E. (2009). What I the issue with disabled student's learning? En M. Fuller, J. Georgeson, M. Healey, A. Hurst, K. Kelly, S. Riddell, ... E. WEEDON (Eds.), Improving disabled students' learning: experiences and outcomes (pp. 3-19). New York: Routledge.

Fundación Universia. (2018). Discapacidad y Universidad. IV Estudio sobre el grado de inclusión del sistema universitario español respecto de la realidad de la discapacidad. Recuperado de https://www.fundacionuniversia.net/wp-content/uploads/2019/05/IVEstudio_UniversidadyDiscapacidad_ACC.pdf

Gale, N., Heath, G., Cameron, E., Rashid, S. y Redwood, S. (2013). Using the framework method for the analysis of qualitative data in multidisciplinary health research. $B M C M e-$ dical Research Methodology, 13(117), 1-8. doi:10.1186/1471-2288-13-117

Gale, T. y Mills, C. (2013). Creating spaces in higher education for marginalized Australians: principles for socially inclusive pedagogies. Enhancing Learning in the Social Sciences, 5(2), 7-19. https://doi.org/10.11120/elss.2013.00008

Gallagher, D., Connor, D. y Ferri, B. (2014). Beyond the far too incessant schism: special education and the social model of disability. International Journal of Inclusive Education, 18(11), 1120-1142. https://doi.org/10.1080/13603116.2013.875599

Garabal-Barbeira, J., Pousada, T., Espinosa, P. C. y Saleta, J. L. (2018). Las actitudes como factor clave en la inclusión universitaria. Revista Española de Discapacidad, 6(1), 181198. doi:10.5569/2340-5104.06.01.09

Gibson, S. (2015). When rights are not enough: what is? Moving towards new pedagogy for inclusive education within UK Universities. International Journal of Inclusive Education, 19(8), 875-886. http://dx.doi.org/10.1080/13603116.2015.1015177 
Hamour, B. (2013). Faculty attitudes toward students with disabilities in a Public University in Jordan. International Education Studies, 6(12), 74-81. doi:10.5539/ies.v6n12p74

Hewett, R., Douglas, G., Mclinden, M. y Keil, S. (2018). Balancing inclusive design, adjustments and personal agency: progressive mutual accommodations and the experiences of university students with vision impairment in the United Kingdom. International Journal of Inclusive Education, 24(2) 1-17. https://doi.org/10.1080/13603116.2018.1492637

Hopkins, S. L., Round, P. N. y Barley, K. D. (2018). Preparing beginning teachers for inclusion: designing and assessing supplementary fieldwork experiences. Teachers and Teaching. Theory and practice, 24(8), 915-930. https://doi.org/10.1080/13540602.2018.1495624

Huber, G. L. (2004). The analysis of qualitative data as process of classification. En M. KieGELMANN (Ed.), Qualitative research in psychology (pp. 206-216). Tübingen: Verlag Ingeborg Huber.

Kioko, V. y Makoelle, T. (2014). Inclusion in Higher Education: learning experiences of disabled students at Winchester University. International Education Studies, 7(6), 106-116. doi:10.5539/ies.v7n6p106

LANGØRgEn, E. y Magnus, E. (2018). We are just ordinary people working hard to reach our goals! Disabled students' participation in Norwegian higher education. Disability and Society, 33(4), 598-617. https://doi.org/10.1080/09687599.2018.1436041

Ley Orgánica 4/2007, de 12 de abril, de universidades. Boletín Oficial del Estado, núm. 89, de 13 de abril de 2007, 16241-16260 . Recuperado de https://www.boe.es/buscar/doc. php?id=BOE-A-2007-7786

Ley Orgánica 3/2018, de 5 de diciembre, de Protección de Datos Personales y garantía de los derechos digitales. Boletín Oficial del Estado, núm. 294, de 6 de abril de 2018, 119788119857. Recuperado de https://www.boe.es/buscar/doc.php?id=BOE-A-2018-16673

Lipka, O., Forkosh, A. y Meer, Y. (2018). Academic support model for post-secondary school students with learning disabilities: student and instructor perceptions. International Journal of Inclusive Education, 23(2), 142-157. doi:10.1080/13603116.2018.1427151

Lombardi, A. R., Murray, C. y Dallas, B. (2013). University faculty attitudes toward disability and inclusive instruction: Comparing two institutions. Journal of Postsecondary Education and Disability, 26(3), 221-232. Recuperado de https://files.eric.ed.gov/fulltext/ EJ1026882.pdf

Lombardi, A., Vukovic, B. y Sala-Bars, I. (2015). International comparisons of inclusive instruction among college faculty in Spain, Canada, and the United States. Journal of Postsecondary Education and Disability, 28(4), 447-460. Recuperado de https://files.eric. ed.gov/fulltext/EJ1093535.pdf

López-Melero, M. (2011). Barreras que impiden la escuela inclusiva y algunas estrategias para construir una escuela sin exclusiones. Innovación Educativa, 21, 37-54. Recuperado de https://minerva.usc.es/xmlui/bitstream/handle/10347/6223/pg_039-056_in21_1. pdf?sequence $=1 \&$ isAllowed $=\mathrm{y}$

Martins, H., Borges, M. y Gonçalves, T. (2018). Attitudes towards inclusion in higher education in a Portuguese university. International Journal of Inclusive Education, 22(5), 527-542. https://doi.org/10.1080/13603116.2017.1377299

Molina, V., Perera, V., Melero, N., Cotán, A. y Moriña, A. (2016). The role of lecturers and inclusive education. Journal of Research in Special Educational Needs, 16(1), 10461049. doi:10.1111/1471-3802.12361 
Moriña, A. y Carballo, R. (2018). Profesorado universitario y educación inclusiva: respondiendo a sus necesidades de formación. Psicologia Escolar e Educacional, SP. Número Especial, 87-95. doi:10.1590/2175-3539/2018/053

Moriña, A., López-Gavira, R. y Molina, V. (2014). Students with disabilities in higher education: a biographical-narrative approach to the role of lecturers. Higher Education Research and Development, 34(1), 147-159. doi:10.1080/07294360.2014.934329

Moriña, A. y Morgado, B. (2018). University surroundings and infrastructures that are accessible and inclusive for all: listening to students with disabilities. Journal of Further and Higher Education, 42(1), 13-23. doi:10.1080/0309877X.2016.118890

Moriña, A., SAndoval, M. y CARnerero, F. (2020). Higher education inclusivity: when the disability enriches the university. Higher Education Research and Development. https:// doi.org/10.1080/07294360.2020.1712676

Mutanga, O. (2018) Inclusion of students with disabilities in South African Higher Education. International Journal of Disability, Development and Education, 65(2), 229-242. doi: 10.1080/1034912X.2017.1368460

Naciones Unidas. (1990). Declaración mundial sobre educación para todos. Recuperado de https://unesdoc.unesco.org/ark:/48223/pf0000127583_spa

Naciones Unidas. (2006). Convención sobre los Derechos de las Personas con Discapacidad. Recuperado de https://www.un.org/disabilities/documents/convention/convoptprot-s. pdf

Oliver, M. y Barnes, C. (2010). Disability studies, disabled people and the struggle for inclusion. British Journal of Sociology of Education, 31(5), 547-560. doi:10.1080/01425692.2 010.500088

Palmer, M. y Harley, D. (2012). Models and measurement in disability: an international review. Health Policy and Planning, 27(5), 357-364. doi:10.1093/heapol/czr047

PAz, E. J. (2018). La formación del profesorado universitario para la atención a la diversidad en la educación superior. IE Revista de Investigación Educativa de la REDIECH, 9(16), 67-82. Recuperado de https://www.redalyc.org/jatsRepo/5216/521654339004/html/index. html

Real Decreto 1720/2007, de 21 de diciembre, por el que se aprueba el Reglamento de desarrollo de la Ley Orgánica 15/1999, de 13 de diciembre, de protección de datos de carácter personal. Boletín Oficial del Estado, núm. 17, de 19 de enero de 2008. Recuperado de https:// www.boe.es/buscar/act.php?id=BOE-A-2008-979

Reupert, A., Hemmings, B. y Connor, J. (2010). Do we practice what we preach? The teaching practices of inclusive educators in tertiary settings. International Journal of Teaching and Learning in Higher Education, 22(2), 120-132.

Riddell, S., Edward, S., Weedon, E. y Ahlgren, L. (2010). Disability and employment statistics. En S. Riddell, S. Edward, E. Weedon y L. Ahlgren (Eds.), Disability, skills and employment. A review of recent statistics and literature on policy and initiatives (pp. 7-37). Manchester: Equality and Human Rigths Commission.

Riddell, S., Tinklin, T. y Wilson, A. (2005). Setting the scene. Disabled students and multiple policy innovations in higher education. En S. Riddell, T. Tinklin y A. Wilson, (Eds.), Disabled students in Higher Education: perspectives on widening access and changing policy (pp. 1-20). New York: Routledge.

Riddell, S. y Weedon, E. (2009). Disabled students and transitions in higher education. En K. Ecclestone, G. Biesta y M. Hughes (Eds.), Transitions and learning through the lifecourse (pp. 103-117). London: Routledge. 
Rojas, S., Haya, I. y Susinos, T. (2016). Growing student voice in curriculum decisions at the university. Journal of Research in Special Educational Needs, 16(1), 563-567. doi:10.1111/1471-3802.12186

Rosa, N., Bogart, K., Bonnett, A., Estill, M. y Colton, C. (2015). Teaching about disability in psychology: an analysis of disability curricula in U.S. Undergraduate Psychology Programs. Teaching of Psychology, 43(1), 59-63. doi:10.1177/0098628315620885

Sanahuja-Gavaldà, J. M., Muñoz-Moreno, J. L. y Gairín-Sallán, J. (2020). Tutoring students with disabilities at the university. Cultura y Educación. https://doi.org/10.1080/113 56405.2019.1709392

Sapon-Shevin, M. (2013). La inclusión real: una perspectiva de justicia social. Revista de Investigación en Educación, 11(3), 71-85.

WeEdon, E. y Riddell, S. (2016). Higher education in Europe: widening participation. En M. Shah, A. Bennett y E. Southgate (Eds.), Widening higher education participation. A global perspective (pp. 49-61). Oxford: Elsevier.

Wolfe, B. y Riggs, E. (2017). Macrosystem analysis of programs and strategies to increase underrepresented populations in the geosciences. Journal of Geoscience Education, 65, 577 593. https://doi.org/10.5408/17-256.1

Yssel, N., PAK, N. y BeILKe, J. (2016). A door must be opened: perceptions of students with disabilities in Higher Education. International Journal of Disability, Development and Education, 63(3), 384-394. http://dx.doi.org/10.1080/1034912X.2015.1123232

Zhang, Y., Rosen, S., Cheng, L. y Li, J. (2018). Inclusive Higher Education for students with disabilities in China: what do the university teachers think? Higher Education Studies, 8(4), 104-115. doi:10.5539/hes.v8n4p104 


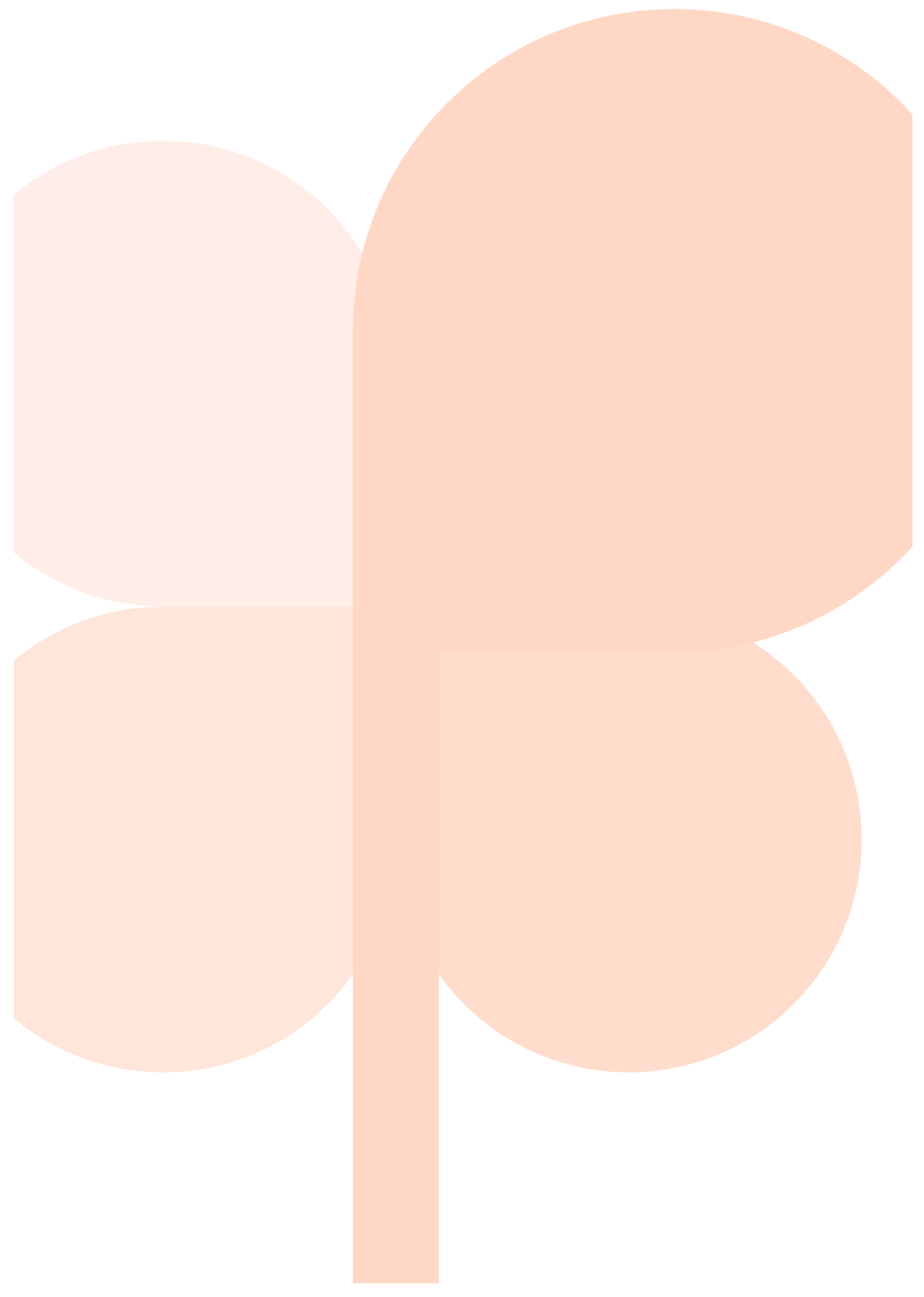

